JAMÅ

Online article and related content current as of April 8, 2009.

\section{Risks and Benefits of Estrogen Plus Progestin in Healthy Postmenopausal Women: Principal Results From the Women's Health Initiative Randomized Controlled Trial}

Writing Group for the Women's Health Initiative Investigators

JAMA. 2002;288(3):321-333 (doi:10.1001/jama.288.3.321)

http://jama.ama-assn.org/cgi/content/full/288/3/321

\section{Correction}

Citations

Related Articles published in the same issue

Related Letters
Contact me if this article is corrected.

This article has been cited 89 times. Contact me when this article is cited.

Failure of Estrogen Plus Progestin Therapy for Prevention Suzanne W. Fletcher et al. JAMA. 2002;288(3):366.

A Woman Attempting to Discontinue Hormone Therapy Robert R. Freedman et al. JAMA. 2002;288(18):2264.

Antioxidants and Risk of Alzheimer Disease Arthur E. Varner et al. JAMA. 2002;288(18):2265

Estrogen Replacement Therapy and Risk of Ovarian Cancer in Postmenopausal Women Kenneth Burry et al. JAMA. 2002;288(20):2538.

Risks of Postmenopausal Hormone Replacement Nina Radford et al. JAMA. 2002;288(22):2819.

Hormone Replacement Following Early Menopause Glinda S. Cooper et al. JAMA. 2002;288(22):2824.

Relationship Between Hormone Replacement Therapy, Socioeconomic Status, and Coronary Heart Disease

Francine Grodstein et al. JAMA. 2003;289(1):44.

Research in Medical Education

Rose Hatala et al. JAMA. 2003;289(2):176.

Estrogen Replacement and Risk of Alzheimer Disease

Amnon Lahad et al. JAMA. 2003;289(9):1100.

Lynn Rosenberg. JAMA. 2003;289(9):1101.

In Reply:

Peter P. Zandi et al. JAMA. 2003;289(9):1101.

In Reply:

Sally A. Shumaker et al. JAMA. 2003;290(13):1707.

Exercise and Risk of Breast Cancer Harry B. Burke. JAMA. 2003;290(24):3193.

Effects of Estrogen-Only Treatment in Postmenopausal Women

Vicki F. Meyer. JAMA. 2004;292(6):683.

Karla Kerlikowske et al. JAMA. 2004:292(6):684.

Katherine L. Brubaker. JAMA. 2004;292(6):686.

Hormone Therapy and Cardiovascular Risk Samuel Shapiro. JAMA. 2007;298(6):623.
Subscribe

http://jama.com/subscribe

Permissions

permissions@ama-assn.org

http://pubs.ama-assn.org/misc/permissions.dt|
Email Alerts

http://jamaarchives.com/alerts

Reprints/E-prints

reprints@ama-assn.org 


\section{Risks and Benefits of Estrogen Plus Progestin in Healthy Postmenopausal Women Principal Results From the Women's Health Initiative Randomized Controlled Trial}

Writing Group for the Women's Health Initiative Investigators

T HE WOMEN'S HEALTH INITIAtive (WHI) focuses on defining the risks and benefits of strategies that could potentially reduce the incidence of heart disease, breast and colorectal cancer, and fractures in postmenopausal women. Between 1993 and 1998, the WHI enrolled 161809 postmenopausal women in the age range of 50 to 79 years into a set of clinical trials (trials of low-fat dietary pattern, calcium and vitamin D supplementation, and 2 trials of postmenopausal hormone use) and an observational study at 40 clinical centers in the United States. ${ }^{1}$ This article reports principal results for the trial of combined estrogen and progestin in women with a uterus. The trial was stopped early based on health risks that exceeded health benefits over an average follow-up of 5.2 years. A parallel trial of estrogen alone in women who have had a hysterectomy is being continued, and the planned end of this trial is March 2005, by which time the average follow-up will be about 8.5 years.

The WHI clinical trials were designed in 1991-1992 using the accumulated evidence at that time. The primary outcome for the trial of estrogen plus progestin was designated as coronary heart disease (CHD). Potential cardioprotection was based on generally

For editorial comment see p 366.
Context Despite decades of accumulated observational evidence, the balance of risks and benefits for hormone use in healthy postmenopausal women remains uncertain.

Objective To assess the major health benefits and risks of the most commonly used combined hormone preparation in the United States.

Design Estrogen plus progestin component of the Women's Health Initiative, a randomized controlled primary prevention trial (planned duration, 8.5 years) in which 16608 postmenopausal women aged 50-79 years with an intact uterus at baseline were recruited by 40 US clinical centers in 1993-1998.

Interventions Participants received conjugated equine estrogens, $0.625 \mathrm{mg} / \mathrm{d}$, plus medroxyprogesterone acetate, $2.5 \mathrm{mg} / \mathrm{d}$, in 1 tablet $(n=8506)$ or placebo $(n=8102)$.

Main Outcomes Measures The primary outcome was coronary heart disease (CHD) (nonfatal myocardial infarction and CHD death), with invasive breast cancer as the primary adverse outcome. A global index summarizing the balance of risks and benefits included the 2 primary outcomes plus stroke, pulmonary embolism (PE), endometrial cancer, colorectal cancer, hip fracture, and death due to other causes.

Results On May 31, 2002, after a mean of 5.2 years of follow-up, the data and safety monitoring board recommended stopping the trial of estrogen plus progestin vs placebo because the test statistic for invasive breast cancer exceeded the stopping boundary for this adverse effect and the global index statistic supported risks exceeding benefits. This report includes data on the major clinical outcomes through April 30, 2002. Estimated hazard ratios (HRs) (nominal 95\% confidence intervals [Cls]) were as follows: CHD, 1.29 (1.02-1.63) with 286 cases; breast cancer, 1.26 (1.00-1.59) with 290 cases; stroke, 1.41 (1.07-1.85) with 212 cases; PE, 2.13 (1.39-3.25) with 101 cases; colorectal cancer, 0.63 (0.43-0.92) with 112 cases; endometrial cancer, 0.83 (0.47-1.47) with 47 cases; hip fracture, 0.66 (0.45-0.98) with 106 cases; and death due to other causes, $0.92(0.74-1.14)$ with 331 cases. Corresponding HRs (nominal 95\% Cls) for composite outcomes were 1.22 (1.09-1.36) for total cardiovascular disease (arterial and venous disease), 1.03 (0.901.17) for total cancer, $0.76(0.69-0.85)$ for combined fractures, $0.98(0.82-1.18)$ for total mortality, and 1.15 (1.03-1.28) for the global index. Absolute excess risks per 10000 personyears attributable to estrogen plus progestin were 7 more CHD events, 8 more strokes, 8 more PEs, and 8 more invasive breast cancers, while absolute risk reductions per 10000 person-years were 6 fewer colorectal cancers and 5 fewer hip fractures. The absolute excess risk of events included in the global index was 19 per 10000 person-years.

Conclusions Overall health risks exceeded benefits from use of combined estrogen plus progestin for an average 5.2-year follow-up among healthy postmenopausal US women. All-cause mortality was not affected during the trial. The risk-benefit profile found in this trial is not consistent with the requirements for a viable intervention for primary prevention of chronic diseases, and the results indicate that this regimen should not be initiated or continued for primary prevention of CHD.

JAMA. 2002;288:321-333

www.jama.com

Author Information and Financial Disclosures appear at the end of this article. 
Figure 1. Profile of the Estrogen Plus Progestin Component of the Women's Health Initiative

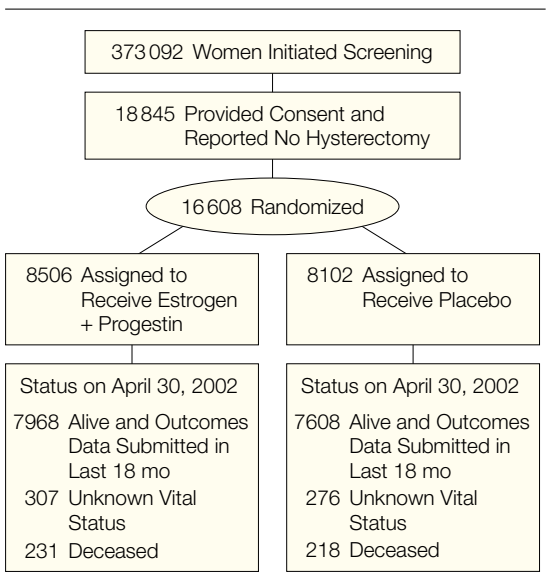

supportive data on lipid levels in intermediate outcome clinical trials, trials in nonhuman primates, and a large body of observational studies suggesting a $40 \%$ to $50 \%$ reduction in risk among users of either estrogen alone or, less frequently, combined estrogen and progestin. ${ }^{2-5}$ Hip fracture was designated as a secondary outcome, supported by observational data as well as clinical trials showing benefit for bone mineral density. ${ }^{6,7}$ Invasive breast cancer was designated as a primary adverse outcome based on observational data. ${ }^{3,8} \mathrm{Ad}-$ ditional clinical outcomes chosen as secondary outcomes that may plausibly be affected by hormone therapy include other cardiovascular diseases; endometrial, colorectal, and other cancers; and other fractures. $3,6,9$

The effect of hormones on overall health was an important consideration in the design and conduct of the WHI clinical trial. In an attempt to summarize important aspects of health benefits vs risks, a global index was defined as the earliest occurrence of CHD, invasive breast cancer, stroke, pulmonary embolism (PE), endometrial cancer, colorectal cancer, hip fracture, or death due to other causes. Compared with total mortality, which may be too insensitive, this index assigns additional weight to the 7 listed diseases. Procedures for monitoring the trial involved semiannual comparisons of the estrogen plus progestin and placebo groups with respect to each of the elements of the global index and to the overall global index.

This report pertains primarily to estrogen plus progestin use among healthy postmenopausal women, since only $7.7 \%$ of participating women reported having had prior cardiovascular disease. During the course of the WHI trial, the Heart and Estrogen/ progestin Replacement Study (HERS) reported its principal results. ${ }^{10}$ HERS was another blinded, randomized controlled trial comparing the same regimen of estrogen plus progestin with placebo among women with a uterus; however, in HERS, all 2763 participating women had documented CHD prior to randomization. The HERS findings of no overall effect on CHD but an apparent increased risk in the first year after randomization seemed surprising given preceding observational studies of hormone use in women with CHD. ${ }^{3}$ Subsequent to HERS, some investigators reanalyzed their observational study data and were able to detect an early elevation in CHD risk among women with prior $\mathrm{CHD}^{11-13}$ but not in ostensibly healthy women, ${ }^{14}$ prompting speculation that any early adverse effect of hormones on CHD incidence was confined to women who have experienced prior CHD events.

The WHI is the first randomized trial to directly address whether estrogen plus progestin has a favorable or unfavorable effect on CHD incidence and on overall risks and benefits in predominantly healthy women.

\section{METHODS \\ Study Population}

Detailed eligibility criteria and recruitment methods have been published. ${ }^{1}$ Briefly, most women were recruited by population-based direct mailing campaigns to age-eligible women, in conjunction with media awareness programs. Eligibility was defined as age 50 to 79 years at initial screening, postmenopausal, likelihood of residence in the area for 3 years, and provision of written informed consent. A woman was considered postmenopausal if she had experienced no vaginal bleeding for 6 months (12 months for 50- to 54-yearolds), had had a hysterectomy, or had ever used postmenopausal hormones. Major exclusions were related to competing risks (any medical condition likely to be associated with a predicted survival of $<3$ years), safety (eg, prior breast cancer, other prior cancer within the last 10 years except nonmelanoma skin cancer, low hematocrit or platelet counts), and adherence and retention concerns (eg, alcoholism, dementia).

A 3-month washout period was required before baseline evaluation of women using postmenopausal hormones at initial screening. Women with an intact uterus at initial screening were eligible for the trial of combined postmenopausal hormones, while women with a prior hysterectomy were eligible for the trial of unopposed estrogen. This report is limited to the 16608 women with an intact uterus at baseline who were enrolled in the trial component of estrogen plus progestin vs placebo. The protocol and consent forms were approved by the institutional review boards for all participating institutions (see Acknowledgment).

\section{Study Regimens, Randomization, and Blinding}

Combined estrogen and progestin was provided in 1 daily tablet containing conjugated equine estrogen (CEE), $0.625 \mathrm{mg}$, and medroxyprogesterone acetate (MPA), $2.5 \mathrm{mg}$ (Prempro, Wyeth Ayerst, Philadelphia, Pa). A matching placebo was provided to the control group. Eligible women were randomly assigned to receive estrogen plus progestin or placebo after eligibility was established and baseline assessments made (FIGURE 1). The randomization procedure was developed at the WHI Clinical Coordinating Center and implemented locally through a distributed study database, using a randomized permuted block algorithm, stratified by clinical center site and age group. All study medication bottles had a unique bottle number and bar code to allow for blinded dispensing. 
Initially, the design allowed women with a uterus to be randomized to receive unopposed estrogen, estrogen plus progestin, or placebo. After the release of the Postmenopausal Estrogen/ Progestin Intervention (PEPI) trial results ${ }^{15}$ indicating that long-term adherence to unopposed estrogen was not feasible in women with a uterus, the WHI protocol was changed to randomize women with a uterus to only estrogen plus progestin or placebo in equal proportions. The 331 women previously randomized to unopposed estrogen were unblinded and reassigned to estrogen plus progestin. These women are included in the estrogen plus progestin group in this report, resulting in 8506 participants in the estrogen plus progestin group vs 8102 in the placebo group. Analysis of the data excluding the women randomized before this protocol change did not affect the results. Considerable effort was made to maintain blinding of other participants and clinic staff. When required for safety or symptom management, an unblinding officer provided the clinic gynecologist, who was not involved with study outcomes activities, with the treatment assignment.

\section{Follow-up}

Study participants were contacted by telephone 6 weeks after randomization to assess symptoms and reinforce adherence. Follow-up for clinical events occurred every 6 months, with annual in-clinic visits required. At each semiannual contact, a standardized interview collected information on designated symptoms and safety concerns, and initial reports of outcome events were obtained using a self-administered questionnaire. Adherence to study interventions was assessed by weighing of returned bottles. The study protocol required annual mammograms and clinical breast examinations; study medications were withheld if safety procedures were not performed, but these participants continued to be followed up. Electrocardiograms were collected at baseline and at follow-up years 3 and 6 .

\section{Data Collection, Management, and Quality Assurance}

All data were collected on standardized study forms by certified staff according to documented study procedures. Study data were entered into a local clinical center database developed and maintained by the Clinical Coordinating Center and provided to each site in the form of a local area network connected to the Clinical Coordinating Center through a wide area network. Data quality was ensured through standard data entry mechanisms, routine reporting and database checks, random chart audits, and routine site visits.

\section{Maintenance/Discontinuation of Study Medications}

During the trial, some flexibility of the dosages of both estrogen and progestin was allowed to manage symptoms such as breast tenderness and vaginal bleeding. Vaginal bleeding was managed according to an algorithm that accounted for the time since randomization, severity of the bleeding, treatment assignment, and endometrial histology. Women who had a hysterectomy after randomization for indications other than cancer were switched to unopposed estrogen or the corresponding placebo without unblinding. These women are included in the original randomization group for analyses.

Permanent discontinuation of study medication was required by protocol for women who developed breast cancer, endometrial pathologic state (hyperplasia not responsive to treatment, atypia, or cancer), deep vein thrombosis (DVT) or PE, malignant melanoma, meningioma, triglyceride level greater than $1000 \mathrm{mg} / \mathrm{dL}$ (11.3 $\mathrm{mmol} / \mathrm{L}$ ), or prescription of estrogen, testosterone, or selective estrogenreceptor modulators by their personal physician. Medications were temporarily discontinued in participants who had acute myocardial infarction (MI), stroke, fracture, or major injury involving hospitalization, surgery involving use of anesthesia, any illness resulting in immobilization for more than 1 week, or any other severe illness in which hormone use is temporarily inappropriate.

\section{Outcome Ascertainment}

Cardiovascular Disease. Coronary heart disease was defined as acute MI requiring overnight hospitalization, silent MI determined from serial electrocardiograms (ECGs), or CHD death. The diagnosis of acute MI was established according to an algorithm adapted from standardized criteria ${ }^{16}$ that included cardiac pain, cardiac enzyme and troponin levels, and ECG readings. The primary analyses included both definite and probable MIs as defined by the algorithm. Myocardial infarction occurring during surgery and aborted MIs were included. An aborted MI was defined as chest pain and ECG evidence of acute MI at presentation, an intervention (eg, thrombolysis) followed by resolution of ECG changes, and all cardiac enzyme levels within normal ranges. Silent MI was diagnosed by comparing baseline and follow-up ECGs at 3 and 6 years after randomization. Coronary death was defined as death consistent with CHD as underlying cause plus 1 or more of the following: preterminal hospitalization with MI within 28 days of death, previous angina or MI and no potentially lethal noncoronary disease, death resulting from a procedure related to coronary artery disease, or death certificate consistent with CHD as the underlying cause. Stroke diagnosis was based on rapid onset of a neurologic deficit lasting more than 24 hours, supported by imaging studies when available. Pulmonary embolism and DVT required clinical symptoms supported by relevant diagnostic studies.

Cancer. Breast, colorectal, endometrial, and other cancers were confirmed by pathological reports when available. Current data indicate that at least $98 \%$ of breast, colorectal, and endometrial cancers and $92 \%$ of other cancers were documented with pathological reports.

Fractures. Reports of hip, vertebral, and other osteoporotic fractures (including all fractures except those of 
Table 1. Baseline Characteristics of the Women's Health Initiative Estrogen Plus Progestin Trial Participants $(\mathrm{N}=16608)$ by Randomization Assignment*

\begin{tabular}{|c|c|c|c|}
\hline Characteristics & $\begin{array}{l}\text { Estrogen + Progestin } \\
\quad(\mathrm{n}=8506)\end{array}$ & $\begin{array}{l}\text { Placebo } \\
(\mathrm{n}=8102)\end{array}$ & $\begin{array}{c}P \\
\text { Value† }\end{array}$ \\
\hline Age at screening, mean (SD), y & $63.2(7.1)$ & $63.3(7.1)$ & .39 \\
\hline $\begin{array}{l}\text { Age group at screening, y } \\
50-59\end{array}$ & 2839 (33.4) & $2683(33.1)]$ & \multirow{3}{*}{.80} \\
\hline $60-69$ & $3853(45.3)$ & $3657(45.1)$ & \\
\hline $70-79$ & $1814(21.3)$ & $1762(21.7)$ & \\
\hline $\begin{array}{l}\text { Race/ethnicity } \\
\text { White }\end{array}$ & 7140 (83.9) & $6805(84.0)^{-}-1-1-1$ & \multirow{6}{*}{.33} \\
\hline Black & 549 (6.5) & $575(7.1)$ & \\
\hline Hispanic & $472(5.5)$ & $416(5.1)$ & \\
\hline American Indian & $26(0.3)$ & $30(0.4)$ & \\
\hline Asian/Pacific Islander & $194(2.3)$ & $169(2.1)$ & \\
\hline Unknown & $125(1.5)$ & $107(1.3)$ & \\
\hline $\begin{array}{l}\text { Hormone use } \\
\text { Never }\end{array}$ & $6280(73.9)$ & $6024(74.4)$ & \multirow{3}{*}{.49} \\
\hline Past & $1674(19.7)$ & $1588(19.6)$ & \\
\hline Currentł & $548(6.4)$ & $487(6.0)$ & \\
\hline $\begin{array}{l}\text { Duration of prior hormone use, y } \\
<5\end{array}$ & $1538(69.1)$ & $1467(70.6)$ & \multirow{3}{*}{.25} \\
\hline $5-10$ & $426(19.1)$ & $357(17.2)$ & \\
\hline$\geq 10$ & $262(11.8)$ & $253(12.2)$ & \\
\hline Body mass index, mean (SD), $\mathrm{kg} / \mathrm{m}^{2} \S$ & $28.5(5.8)$ & $28.5(5.9)$ & .66 \\
\hline $\begin{array}{l}\text { Body mass index, } \mathrm{kg} / \mathrm{m}^{2} \\
<25\end{array}$ & $2579(30.4)$ & $2479(30.8)$ & \multirow{3}{*}{.89} \\
\hline $25-29$ & 2992 (35.3) & $2834(35.2)$ & \\
\hline$\geq 30$ & 2899 (34.2) & $2737(34.0)$ & \\
\hline Systolic BP, mean (SD), mm Hg & $127.6(17.6)$ & $127.8(17.5)$ & .51 \\
\hline Diastolic BP, mean (SD), mm Hg & $75.6(9.1)$ & $75.8(9.1)$ & .31 \\
\hline $\begin{array}{l}\text { Smoking } \\
\text { Never }\end{array}$ & 4178 (49.6) & 3999 (50.0) & \multirow{3}{*}{.85} \\
\hline Past & 3362 (39.9) & $3157(39.5)$ & \\
\hline Current & $880(10.5)$ & $838(10.5)$ & \\
\hline $\begin{array}{l}\text { Parity } \\
\quad \text { Never pregnant/no term pregnancy }\end{array}$ & $856(10.1)$ & $832(10.3)-7$ & \multirow{2}{*}{.67} \\
\hline$\geq 1$ term pregnancy & 7609 (89.9) & $7233(89.7)$ & \\
\hline $\begin{array}{l}\text { Age at first birth, y\| } \\
<20\end{array}$ & $1122(16.4)$ & $1114(17.4)$ & \multirow{3}{*}{.11} \\
\hline $20-29$ & 4985 (73.0) & $4685(73.0)$ & \\
\hline$\geq 30$ & $723(10.6)$ & $621(9.7)$ & \\
\hline Treated for diabetes & $374(4.4)$ & $360(4.4)$ & .88 \\
\hline $\begin{array}{c}\text { Treated for hypertension or } \\
\mathrm{BP} \geq 140 / 90 \mathrm{~mm} \mathrm{Hg}\end{array}$ & 3039 (35.7) & 2949 (36.4) & .37 \\
\hline $\begin{array}{l}\text { Elevated cholesterol levels requiring } \\
\text { medication }\end{array}$ & $944(12.5)$ & $962(12.9)$ & .50 \\
\hline Statin use at baseline & $590(6.9)$ & $548(6.8)$ & .66 \\
\hline Aspirin use $(\geq 80 \mathrm{mg} / \mathrm{d}$ ) at baseline & $1623(19.1)$ & $1631(20.1)$ & .09 \\
\hline History of myocardial infarction & $139(1.6)$ & $157(1.9)$ & .14 \\
\hline History of angina & $238(2.8)$ & $234(2.9)$ & .73 \\
\hline History of CABG/PTCA & $95(1.1)$ & $120(1.5)$ & .04 \\
\hline History of stroke & $61(0.7)$ & $77(1.0)$ & .10 \\
\hline History of DVT or PE & $79(0.9)$ & $62(0.8)$ & .25 \\
\hline Female relative had breast cancer & $1286(16.0)$ & 1175 (15.3) & .28 \\
\hline Fracture at age $\geq 55 \mathrm{y}$ & $1031(13.5)$ & 1029 (13.6) & .87 \\
\hline
\end{tabular}

the ribs, chest/sternum, skull/face, fingers, toes, and cervical vertebrae) were routinely ascertained. All fracture outcomes were verified by radiology reports. Study radiographs were not obtained to ascertain subclinical vertebral fractures.

This report is based on outcomes adjudicated by clinical center physician adjudicators, as used for trialmonitoring purposes. Clinical center physician adjudicators were centrally trained and blinded to treatment assignment and participants' symptoms. Future communications will report results based on centrally adjudicated outcomes and will include a broader range of outcomes with more extensive explanatory analyses. Since this report is presented before the planned study closeout, outcome information is still being collected and adjudicated. Local adjudication is complete for approximately $96 \%$ of the designated selfreported events. To date, agreement rates between local and central adjudication are: MI, 84\%; revascularization procedures, 97\%; PE, 89\%; DVT, $84 \%$; stroke, $94 \%$; invasive breast cancer, $98 \%$; endometrial cancer, $96 \%$; colorectal cancer, $98 \%$; hip fracture, $95 \%$; and specific cause of death, $82 \%$. When related cardiovascular conditions are combined (eg, when unstable angina or congestive heart failure is grouped with MI), agreement rates exceed $94 \%$ for cardiovascular disease and $90 \%$ for specific cause of death.

\section{Statistical Analyses}

All primary analyses use time-toevent methods and are based on the intention-to-treat principle. For a given outcome, the time of event was defined as the number of days from randomization to the first postrandomization diagnosis, as determined by the local adjudicator. For silent MIs, the date of the follow-up ECG applied. Participants without a diagnosis were censored for that event at the time of last follow-up contact. Primary outcome comparisons are presented as hazard ratios (HRs) and 95\% confidence intervals (CIs) from Cox proportional haz- 
ards analyses, ${ }^{17}$ stratified by clinical center, age, prior disease, and randomization status in the low-fat diet trial.

Two forms of CIs are presented, nominal and adjusted. Nominal 95\% CIs describe the variability in the estimates that would arise from a simple trial for a single outcome. Although traditional, these CIs do not account for the multiple statistical testing issues (across time and across outcome categories) that occurred in this trial, so the probability is greater than .05 that at least 1 of these CIs will exclude unity under an overall null hypothesis. The adjusted 95\% CIs presented herein use group sequential methods to correct for multiple analyses over time. A Bonferroni correction for 7 outcomes as specified in the monitoring plan (described herein) was applied to all clinical outcomes other than CHD and breast cancer, the designated primary and primary adverse effect outcomes, and the global index. The adjusted CIs are closely related to the monitoring procedures and, as such, represent a conservative assessment of the evidence. This report focuses primarily on results using the unadjusted statistics and also relies on consistency across diagnostic categories, supportive data from other studies, and biologic plausibility for interpretation of the findings.

\section{Data and Safety Monitoring}

Trial monitoring guidelines for early stopping considerations were based on O'Brien-Fleming boundaries ${ }^{18}$ using asymmetric upper and lower boundaries: a 1-sided, .025-level upper boundary for benefit and 1-sided, .05-level lower boundaries for adverse effects. The adverse-effect boundaries were further adjusted with a Bonferroni correction for the 7 major outcomes other than breast cancer that were specifically monitored (CHD, stroke, PE, colorectal cancer, endometrial cancer, hip fracture, and death due to other causes). The global index of monitored outcomes played a supportive role as a summary measure of the overall balance of risks and benefits. Trial monitoring for early stopping consider-

Table 1. Baseline Characteristics of the Women's Health Initiative Estrogen Plus Progestin Trial Participants ( $\mathrm{N}=16$ 608) by Randomization Assignment* (cont)

\begin{tabular}{|c|c|c|c|}
\hline Characteristics & $\begin{array}{l}\text { Estrogen + Progestin } \\
(n=8506)\end{array}$ & $\begin{array}{l}\text { Placebo } \\
(n=8102)\end{array}$ & $\begin{array}{c}P \\
\text { Value }\end{array}$ \\
\hline \multicolumn{3}{|l|}{$\begin{array}{l}\text { Gail model 5-year risk of breast cancer, \% } \\
<1\end{array}$} & \multirow{4}{*}{.64} \\
\hline $1-<2$ & 5384 (63.3) & $5139(63.4)$ & \\
\hline $2-<5$ & $1751(20.6)$ & $1621(20.0)$ & \\
\hline$\geq 5$ & $81(1.0)$ & $71(0.9)$ & \\
\hline \multicolumn{3}{|l|}{ No. of falls in last $12 \mathrm{mo}$} & \multirow{4}{*}{.18} \\
\hline 1 & $1643(21.0)$ & $1545(20.2)$ & \\
\hline 2 & $651(8.3)$ & $645(8.4)$ & \\
\hline$\geq 3$ & $349(4.5)$ & $303(4.0)$ & \\
\hline \multicolumn{4}{|c|}{$\begin{array}{l}\text { *Data are presented as number (percentage) of patients unless otherwise noted. BP indicates blood pressure; CABG/ } \\
\text { PTCA, coronary artery bypass graft/percutaneous transluminal coronary angioplasty; DVT, deep vein thrombosis; } \\
\text { and PE, pulmonary embolism. } \\
\text { †Based on } \chi^{2} \text { tests (categorical variables) or } t \text { tests (continuous variables). } \\
\text { tRequired a 3-month washout prior to randomization. } \\
\text { \$Total number of participants with data available was } 8470 \text { for estrogen plus progestin and } 8050 \text { for placebo. } \\
\text { ॥Among women who reported having a term pregnancy. } \\
\text { IStatins are 3-hydroxy-3-methylglutaryl coenzyme A reductase inhibitors. }\end{array}$} \\
\hline
\end{tabular}

ations was conducted semiannually by an independent data and safety monitoring board (DSMB). Aspects of the monitoring plan have been published. ${ }^{19}$

\section{RESULTS \\ Trial Monitoring and Early Stopping}

Formal monitoring began in the fall of 1997 with the expectation of final analysis in 2005 after an average of approximately 8.5 years of follow-up. Late in 1999 , with 5 interim analyses completed, the DSMB observed small but consistent early adverse effects in cardiovascular outcomes and in the global index. None of the disease-specific boundaries had been crossed. In the spring of 2000 and again in the spring of 2001, at the direction of the DSMB, hormone trial participants were given information indicating that increases in MI, stroke, and PE/DVT had been observed and that the trial continued because the balance of risks and benefits remained uncertain.

In reviewing the data for the 10th interim analyses on May 31, 2002, the DSMB found that the adverse effects in cardiovascular diseases persisted, although these results were still within the monitoring boundaries. However, the design-specified weighted log-rank test statistic for breast cancer $(z=-3.19)$ crossed the designated boundary $(z=-2.32)$ and the global index was supportive of a finding of overall harm $(z=-1.62)$. Updated analyses including 2 months of additional data, available by the time of the meeting, did not appreciably change the overall results. On the basis of these data, the DSMB concluded that the evidence for breast cancer harm, along with evidence for some increase in CHD, stroke, and PE, outweighed the evidence of benefit for fractures and possible benefit for colon cancer over the average 5.2-year follow-up period. Therefore, the DSMB recommended early stopping of the estrogen plus progestin component of the trial. Because the balance of risks and benefits in the unopposed-estrogen component remains uncertain, the DSMB recommended continuation of that component of the WHI. Individual trial participants have been informed.

\section{Baseline Characteristics}

There were no substantive differences between study groups at baseline; 8506 women were randomized into the estrogen plus progestin group and 8102 into the placebo group (TABLE 1). The mean (SD) age was 63.3 (7.1) years. Two thirds of the women who reported prior or current hormone use had taken combined hormones and one third had used unopposed estrogen. 
Figure 2. Cumulative Dropout and Drop-in Rates by Randomization Assignment and Follow-up Duration

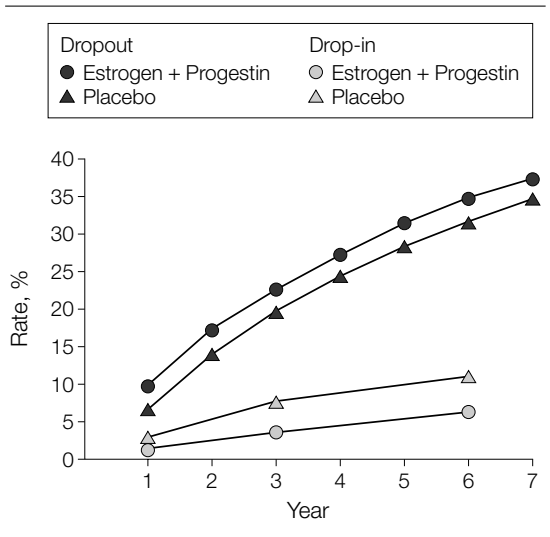

Dropout refers to women who discontinued study medication; drop-in, women who discontinued study medication and received postmenopausal hormones through their own clinician.
Prevalence of prior cardiovascular disease was low and levels of cardiovascular risk factors were consistent with a generally healthy population of postmenopausal women. An assessment of commonly studied breast cancer risk factors, both individually and combined using the Gail model, ${ }^{20}$ indicate that the cohort in general was not at increased risk of breast cancer.

\section{Follow-up, Adherence, and Unblinding}

Vital status is known for 16025 randomized participants (96.5\%), including $449(2.7 \%)$ known to be deceased. A total of 583 (3.5\%) participants were lost to follow-up or stopped providing outcomes information for more than 18 months. The remaining 15576 (93.8\%) provided recent outcome information (Figure 1).

At the time of this report, all women had been enrolled for at least 3.5 years, with an average follow-up of 5.2 years and a maximum of 8.5 years. A substantial number of women had stopped taking study drugs at some time $(42 \%$ of estrogen plus progestin and 38\% of placebo). Dropout rates over time (FIGURE 2) exceeded design projections, particularly early on, but compare favorably with community-based adherence to postmenopausal hormones. ${ }^{21}$ Some women in both groups initiated hormone use through their own clinician $(6.2 \%$ in the estrogen plus progestin group and $10.7 \%$ in the placebo group cumulatively by the sixth

Table 2. Clinical Outcomes by Randomization Assignment*

\begin{tabular}{|c|c|c|c|c|c|}
\hline \multirow[b]{2}{*}{ Outcomes } & \multicolumn{2}{|c|}{ No. of Patients (Annualized \%) } & \multirow[b]{2}{*}{ Hazard Ratio } & \multirow[b]{2}{*}{ Nominal 95\% Cl } & \multirow[b]{2}{*}{ Adjusted $95 \% \mathrm{Cl}$} \\
\hline & $\begin{array}{l}\text { Estrogen + Progestin } \\
\quad(n=8506)\end{array}$ & $\begin{array}{l}\text { Placebo } \\
(\mathrm{n}=8102)\end{array}$ & & & \\
\hline Follow-up time, mean (SD), mo & $62.2(16.1)$ & $61.2(15.0)$ & NA & NA & NA \\
\hline \multicolumn{6}{|l|}{ Cardiovascular disease $\dagger$} \\
\hline $\mathrm{CHD}$ & $164(0.37)$ & $122(0.30)$ & 1.29 & $1.02-1.63$ & $0.85-1.97$ \\
\hline CHD death & $33(0.07)$ & $26(0.06)$ & 1.18 & $0.70-1.97$ & $0.47-2.98$ \\
\hline Nonfatal Ml & $133(0.30)$ & $96(0.23)$ & 1.32 & $1.02-1.72$ & $0.82-2.13$ \\
\hline CABG/PTCA & $183(0.42)$ & $171(0.41)$ & 1.04 & $0.84-1.28$ & $0.71-1.51$ \\
\hline Stroke & $127(0.29)$ & $85(0.21)$ & 1.41 & $1.07-1.85$ & $0.86-2.31$ \\
\hline Fatal & $16(0.04)$ & $13(0.03)$ & 1.20 & $0.58-2.50$ & $0.32-4.49$ \\
\hline Nonfatal & $94(0.21)$ & $59(0.14)$ & 1.50 & $1.08-2.08$ & $0.83-2.70$ \\
\hline Venous thromboembolic disease & $151(0.34)$ & $67(0.16)$ & 2.11 & $1.58-2.82$ & $1.26-3.55$ \\
\hline Deep vein thrombosis & $115(0.26)$ & $52(0.13)$ & 2.07 & $1.49-2.87$ & $1.14-3.74$ \\
\hline Pulmonary embolism & $70(0.16)$ & $31(0.08)$ & 2.13 & $1.39-3.25$ & $0.99-4.56$ \\
\hline Total cardiovascular disease & $694(1.57)$ & $546(1.32)$ & 1.22 & $1.09-1.36$ & $1.00-1.49$ \\
\hline$\frac{\text { Invasive breast }}{\text { Endometrial }}$ & $\begin{array}{r}166(0.38) \\
22(0.05)\end{array}$ & $\begin{array}{r}124(0.30) \\
25(0.06)\end{array}$ & $\begin{array}{l}1.26 \\
0.83\end{array}$ & $\begin{array}{l}1.00-1.59 \\
0.47-1.47\end{array}$ & $\begin{array}{l}0.83-1.92 \\
0.29-2.32\end{array}$ \\
\hline Colorectal & $45(0.10)$ & $67(0.16)$ & 0.63 & $0.43-0.92$ & $0.32-1.24$ \\
\hline Total & $502(1.14)$ & $458(1.11)$ & 1.03 & $0.90-1.17$ & $0.86-1.22$ \\
\hline \multicolumn{6}{|l|}{ Fractures } \\
\hline Hip & $44(0.10)$ & $62(0.15)$ & 0.66 & $0.45-0.98$ & $0.33-1.33$ \\
\hline Vertebral & $41(0.09)$ & $60(0.15)$ & 0.66 & $0.44-0.98$ & $0.32-1.34$ \\
\hline Other osteoporotic $\neq$ & $579(1.31)$ & $701(1.70)$ & 0.77 & $0.69-0.86$ & $0.63-0.94$ \\
\hline Total & $650(1.47)$ & $788(1.91)$ & 0.76 & $0.69-0.85$ & $0.63-0.92$ \\
\hline \multicolumn{6}{|l|}{ Death } \\
\hline Due to other causes & $165(0.37)$ & $166(0.40)$ & 0.92 & $0.74-1.14$ & $0.62-1.35$ \\
\hline Total & $231(0.52)$ & $218(0.53)$ & 0.98 & $0.82-1.18$ & $0.70-1.37$ \\
\hline Global index§ & $751(1.70)$ & $623(1.51)$ & 1.15 & $1.03-1.28$ & $0.95-1.39$ \\
\hline \multicolumn{6}{|c|}{$\begin{array}{l}\text { *Cl indicates confidence interval; NA, not applicable; CHD, coronary heart disease; MI, myocardial infarction; CABG, coronary artery bypass grafting; and PTCA, percutaneous } \\
\text { transluminal coronary angioplasty. } \\
\text { †CHD includes acute Ml requiring hospitalization, silent MI determined from serial electrocardiograms, and coronary death. There were } 8 \text { silent Mls. Total cardiovascular disease is } \\
\text { limited to events during hospitalization except venous thromboembolic disease reported after January } 1,2000 \text {. } \\
\text { fOther osteoporotic fractures include all fractures other than chest/sternum, skull/face, fingers, toes, and cervical vertebrae, as well as hip and vertebral fractures reported sepa- } \\
\text { rately. } \\
\text { §The global index represents the first event for each participant from among the following types: CHD, stroke, pulmonary embolism, breast cancer, endometrial cancer, colorectal } \\
\text { cancer, hip fracture, and death due to other causes. }\end{array}$} \\
\hline
\end{tabular}


year). These "drop-in" rates were also greater than expected.

At the time of this report, clinic gynecologists had been unblinded to treatment assignment for 3444 women in the estrogen plus progestin group and 548 women in the placebo group, primarily to manage persistent vaginal bleeding. During the trial, 248 women in the estrogen plus progestin group and 183 in the placebo group had a hysterectomy.

\section{Intermediate Cardiovascular Disease End Points}

Blood lipid levels, assessed in an 8.6\% subsample of fasting blood specimens collected from women at baseline and year 1 , showed greater reductions in low-density lipoprotein cholesterol $(-12.7 \%)$ and increases in highdensity lipoprotein cholesterol (7.3\%) and triglycerides (6.9\%) with estrogen plus progestin relative to placebo (data not shown), consistent with HERS and PEPI. ${ }^{10,22}$ Systolic blood pressure was, on average, $1.0 \mathrm{~mm} \mathrm{Hg}$ higher in women taking estrogen plus progestin at 1 year, rising to $1.5 \mathrm{~mm} \mathrm{Hg}$ at 2 years and beyond (data not shown). Diastolic blood pressures did not differ.

\section{Clinical Outcomes}

Cardiovascular Disease. Overall CHD rates were low (TABLE 2 ). The rate of women experiencing CHD events was increased by $29 \%$ for women taking estrogen plus progestin relative to placebo (37 vs 30 per 10000 personyears), reaching nominal statistical significance (at the .05 level). Most of the excess was in nonfatal MI. No significant differences were observed in CHD deaths or revascularization procedures (coronary artery bypass grafting or percutaneous transluminal coronary angioplasty). Stroke rates were also higher in women receiving estrogen plus progestin ( $41 \%$ increase; 29 vs 21 per 10000 person-years), with most of the elevation occurring in nonfatal events. Women in the estrogen plus progestin group had 2 -fold greater rates of venous thromboembolism (VTE), as well as DVT and PE individually, with almost all associated CIs excluding 1.

\begin{tabular}{|c|c|c|}
\hline & \multicolumn{2}{|c|}{ No. (Annualized \%) } \\
\hline & Estrogen + Progestin $(n=8506)$ & Placebo $(n=8102)$ \\
\hline Total deaths & $231(0.52)$ & $218(0.53)$ \\
\hline Adjudicated deaths & $215(0.49)$ & $201(0.49)$ \\
\hline Cardiovascular & $65(0.15)$ & $55(0.13)$ \\
\hline Breast cancer & $3(0.01)$ & $2(<0.01)$ \\
\hline Other cancer & $104(0.24)$ & $86(0.21)$ \\
\hline Other known cause & $34(0.08)$ & $41(0.10)$ \\
\hline Unknown cause & $9(0.02)$ & $17(0.04)$ \\
\hline
\end{tabular}

Rates of VTE were 34 and 16 per 10000 person-years in the estrogen plus progestin and placebo groups, respectively. Total cardiovascular disease, including other events requiring hospitalization, was increased by $22 \%$ in the estrogen plus progestin group.

Cancer. The invasive breast cancer rates in the placebo group were consistent with design expectations. The $26 \%$ increase (38 vs 30 per 10000 person-years) observed in the estrogen plus progestin group almost reached nominal statistical significance and, as noted herein, the weighted test statistic used for monitoring was highly significant. No significant difference was observed for in situ breast cancers. Follow-up rates for mammography were comparable in the estrogen plus progestin and placebo groups. Colorectal cancer rates were reduced by $37 \%$ ( 10 vs 16 per 10000 person-years), also reaching nominal statistical significance. Endometrial cancer incidence was not affected, nor was lung cancer incidence (54 vs 50; HR, 1.04; 95\% CI, 0.71-1.53) or total cancer incidence.

Fractures. This cohort experienced low hip fracture rates (10 per 10000 person-years in the estrogen plus progestin group vs 15 per 10000 personyears in the placebo group). Estrogen plus progestin reduced the observed hip and clinical vertebral fracture rates by one third compared with placebo, both nominally significantly. The reductions in other osteoporotic fractures (23\%) and total fractures (24\%) were statistically significant (all associated CIs exclude 1).

The global index showed a nominally significant $15 \%$ increase in the es- trogen plus progestin group (170 vs 151 per 10000 person-years). There were no differences in mortality or cause of death between groups (TABLE 3).

\section{Time Trends}

The Kaplan Meier estimates of cumulative hazards (FIGURE 3) for CHD indicate that the difference between treatment groups began to develop soon after randomization. These curves provide little evidence of convergence through 6 years of follow-up. The cumulative hazards for stroke begin to diverge between 1 and 2 years after randomization, and this difference persists beyond the fifth year. For PE, the curves separate soon after randomization and show continuing adverse effects throughout the observation period. For breast cancer, the cumulative hazard functions are comparable through the first 4 years, at which point the curve for estrogen plus progestin begins to rise more rapidly than that for placebo. Curves for colorectal cancer show benefit beginning at 3 years, and curves for hip fracture show increasing cumulative benefit over time. The difference in hazard rates for the global index (FIGURE 4) suggests a gradual increase in adverse effects compared with benefits for estrogen plus progestin through year 5 , with a possible narrowing of the difference by year 6 ; however, HR estimates tend to be unstable beyond 6 years after randomization. Total mortality rates are indistinguishable between estrogen plus progestin and placebo.

Tests for linear trends with time since randomization, based on a Cox proportional hazards model with a time- 
dependent covariate, detected no trend with time for CHD, stroke, colorectal cancer, hip fracture, total mortality, or the global index (TABLE 4). There was some evidence for an increasing risk of breast cancer over time with estrogen plus progestin $(z=2.56$ compared with a nominal $z$ score for statistical signifi-

Figure 3. Kaplan-Meier Estimates of Cumulative Hazards for Selected Clinical Outcomes

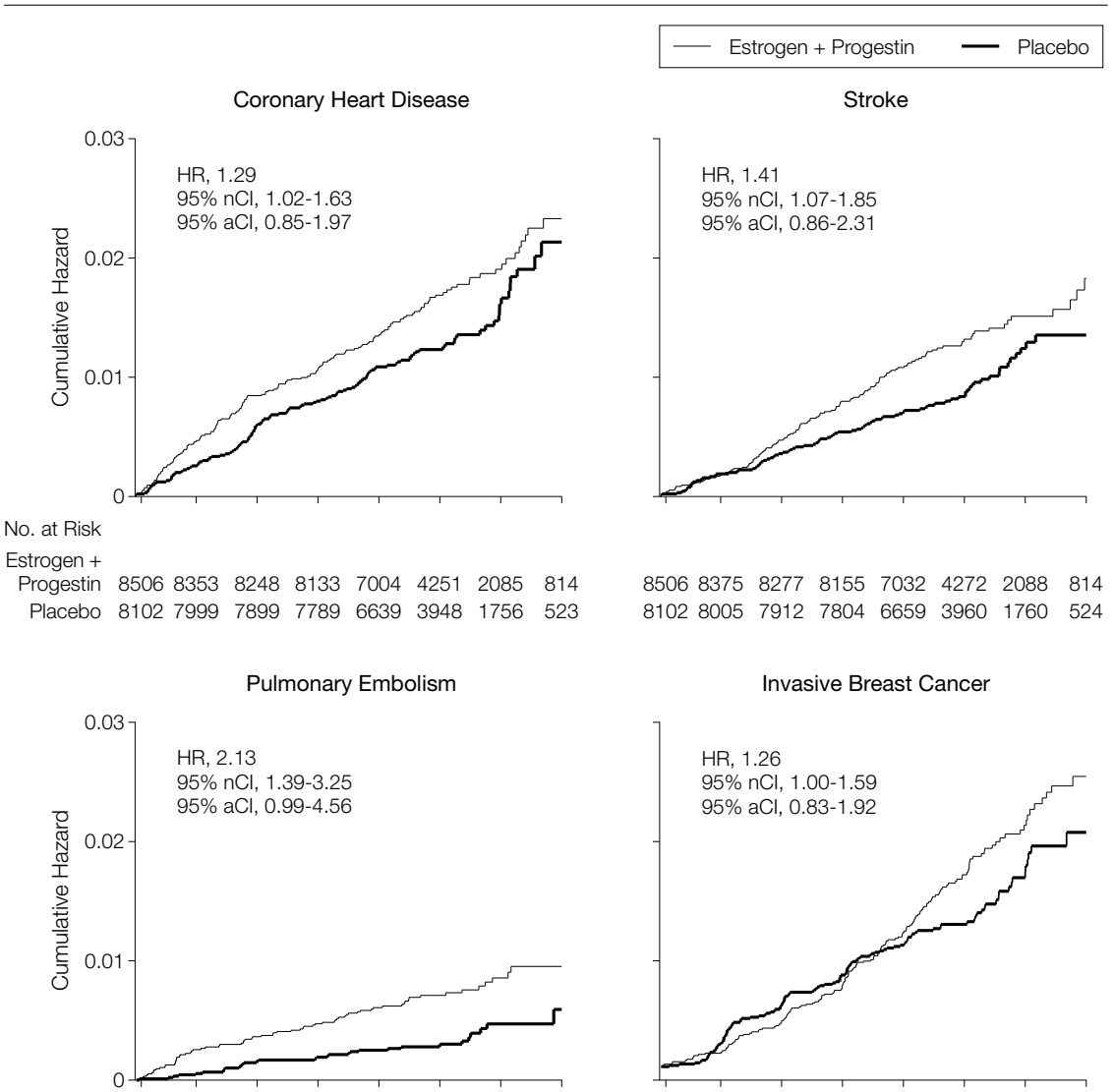

No. at Risk

Estrogen +

Progestin $8506 \quad 8364 \quad 8280 \quad 8174 \quad 7054 \quad 4295 \quad 2108 \quad 820$ Placebo $81028013 \quad 7924 \quad 782566793973 \quad 1770 \quad 526$

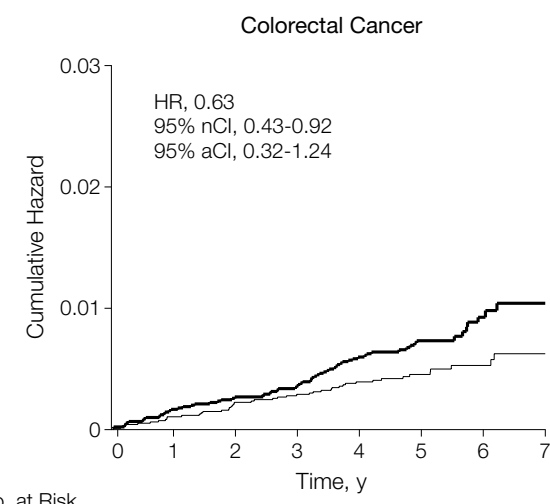

No. at Risk

Estrogen +

Progestin $8506 \quad 83798297 \quad 8194 \quad 7073 \quad 4305 \quad 2111 \quad 825$ Placebo $81028003 \quad 7916781466603958 \quad 1756 \quad 522$

$\mathrm{HR}$ indicates hazard ratio; $\mathrm{nCl}$, nominal confidence interval; and $\mathrm{aCl}$, adjusted confidence interval.

cance of 1.96) and a decreasing risk of VTE with time $(z=-2.45)$. These results must be viewed cautiously because the number of events in each interval is modest, the data in later years are still incomplete, and later year comparisons are limited to women still at risk of their first event for that outcome.

\section{Subgroup Analyses}

Cardiovascular Disease. A small subset of women ( $n=400$; average followup, 57.4 months) in WHI reported conditions at baseline that would have made them eligible for HERS, ie, prior MI or revascularization procedures. Among these women with established coronary disease, the HR for subsequent CHD for estrogen plus progestin relative to placebo was 1.28 (95\% CI, 0.64-2.56) with 19 vs 16 events. The remaining women, those without prior CHD, had an identical HR for CHD (145 vs 106; HR, 1.28; 95\% CI, 1.00$1.65)$. Few women with a history of VTE were enrolled, but these data suggest a possibility that these women may be at greater risk of future VTE events when taking estrogen plus progestin (7 vs 1 ; $\mathrm{HR}, 4.90$; 95\% CI, 0.58-41.06) than those without a history of VTE (144 vs 66 ; HR, 2.06; 95\% CI, 1.54-2.76). For stroke, prior history did not confer additional risk ( 1 vs 5 in women with prior stroke; HR, 0.46; $95 \%$ CI, $0.05-4.51 ; 126$ vs 80 with no prior stroke; HR, 1.47 ; 95\% CI, 1.11-1.95). No noteworthy interactions with age, race/ethnicity, body mass index, prior hormone use, smoking status, blood pressure, diabetes, aspirin use, or statin use were found for the effect of estrogen plus progestin on CHD, stroke, or VTE.

Breast Cancer. Women reporting prior postmenopausal hormone use had higher HRs for breast cancer associated with estrogen plus progestin use than those who never used postmenopausal hormones (among never users, 114 vs 102 ; HR, 1.06; $95 \% \mathrm{CI}$, 0.81-1.38; for women with $<5$ years of prior use, 32 vs 15; HR, 2.13; 95\% CI, 1.15-3.94; for women with 5-10 
years of prior use, 11 vs 2; HR, 4.61; 95\% CI, 1.01-21.02; and for women with $\geq 10$ years of prior use, 9 vs 5 ; HR, 1.81; 95\% CI, 0.60-5.43; test for trend, $z=2.17$ ). No interactions between estrogen plus progestin and age, race/ethnicity, family history, parity, age at first birth, body mass index, or Gail-model risk score were observed for invasive breast cancer.

\section{Further Analyses}

Because a number of women stopped study medications during follow-up, several analyses were performed to examine the sensitivity of the principal HR estimates to actual use of study medications. Analyses that censored a woman's event history 6 months after becoming nonadherent (using $<80 \%$ of or stopping study drugs) produced the largest changes to estimated effect sizes. This approach increased HRs to 1.51 for CHD, to 1.49 for breast cancer, to 1.67 for stroke, and to 3.29 for VTE. Analyses attributing events to actual hor- mone use ("as treated," allowing for a 6-month lag) produced more modest changes to these estimates. Analyses excluding women randomized during the period when the unopposed-estrogen component was open to women with a uterus and analyses stratifying by enrollment period did not substantially

Figure 4. Kaplan-Meier Estimates of Cumulative Hazards for Global Index and Death

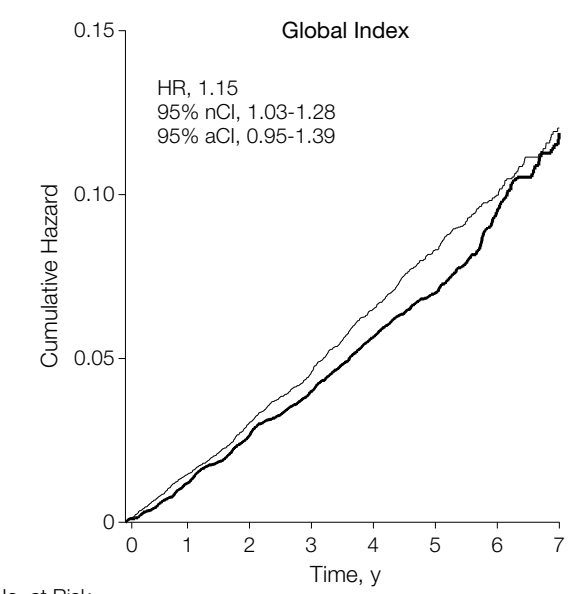

No. at Risk

Estrogen

Progestin $85068291 \quad 8113 \quad 7927 \quad 6755 \quad 4058 \quad 1964 \quad 758$ Placebo $810279397774 \quad 7607 \quad 6425 \quad 3794 \quad 1662 \quad 495$

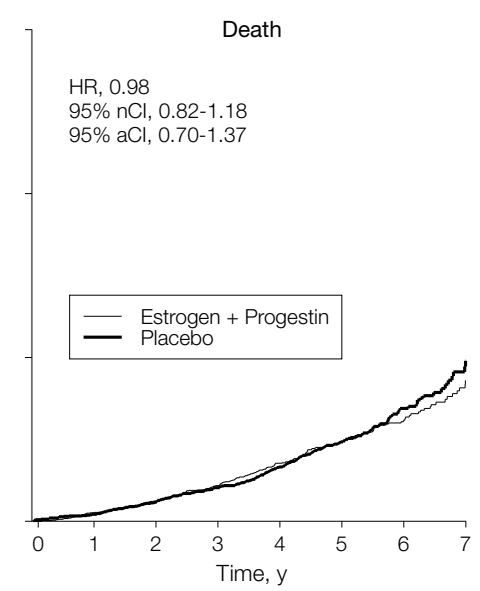

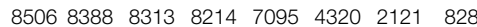

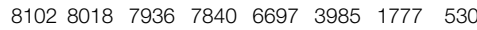

$\mathrm{HR}$ indicates hazard ratio; $\mathrm{nCl}$, nominal confidence interval; and $\mathrm{aCl}$, adjusted confidence interval.

\begin{tabular}{|c|c|c|c|c|c|c|c|c|c|c|}
\hline \multirow[b]{2}{*}{ Outcomes } & \multicolumn{3}{|c|}{ Year 1} & \multicolumn{3}{|c|}{ Year 2} & \multicolumn{3}{|c|}{ Year 3} & \\
\hline & $E+P$ & Placebo & Ratio & $E+P$ & Placebo & Ratio & $E+P$ & Placebo & Ratio & \\
\hline No. of participant-years & 8435 & 8050 & & 8353 & 7980 & & 8268 & 7888 & & \\
\hline Coronary heart disease & $43(0.51)$ & $23(0.29)$ & 1.78 & $36(0.43)$ & $30(0.38)$ & 1.15 & $20(0.24)$ & $18(0.23)$ & 1.06 & \\
\hline Stroke & $17(0.20)$ & $17(0.21)$ & 0.95 & $27(0.32)$ & $15(0.19)$ & 1.72 & $30(0.36)$ & $16(0.20)$ & 1.79 & \\
\hline Venous thromboembolism & $49(0.58)$ & $13(0.16)$ & 3.60 & $26(0.31)$ & $11(0.14)$ & 2.26 & $21(0.25)$ & $12(0.15)$ & 1.67 & \\
\hline Invasive breast cancer & $11(0.13)$ & $17(0.21)$ & 0.62 & $26(0.31)$ & $30(0.38)$ & 0.83 & $28(0.34)$ & $23(0.29)$ & 1.16 & \\
\hline Endometrial cancer & $2(0.02)$ & $2(0.02)$ & 0.95 & $4(0.05)$ & $4(0.05)$ & 0.96 & $4(0.05)$ & $5(0.06)$ & 0.76 & \\
\hline Colorectal cancer & $10(0.12)$ & $15(0.19)$ & 0.64 & $11(0.13)$ & $9(0.11)$ & 1.17 & $6(0.07)$ & $8(0.10)$ & 0.72 & \\
\hline Hip fracture & $6(0.07)$ & $9(0.11)$ & 0.64 & $8(0.10)$ & $13(0.16)$ & 0.59 & $11(0.13)$ & $12(0.15)$ & 0.87 & \\
\hline Total death & $22(0.26)$ & $17(0.21)$ & 1.24 & $30(0.36)$ & $30(0.38)$ & 0.96 & $39(0.47)$ & $35(0.44)$ & 1.06 & \\
\hline \multirow[t]{2}{*}{ Global index } & $123(1.46)$ & $96(1.19)$ & 1.22 & $134(1.60)$ & $117(1.47)$ & 1.09 & $127(1.54)$ & $107(1.36)$ & 1.13 & \\
\hline & \multicolumn{3}{|c|}{ Year 4} & \multicolumn{3}{|c|}{ Year 5} & \multicolumn{3}{|c|}{ Year 6 and Later } & \multirow{2}{*}{$\begin{array}{c}z \text { Score } \\
\text { for Trend } \dagger\end{array}$} \\
\hline Outcomes & $E+P$ & Placebo & Ratio & $E+P$ & Placebo & Ratio & $E+P$ & Placebo & Ratio & \\
\hline No. of participant-years & 7926 & 7562 & & 5964 & 5566 & & 5129 & 4243 & & \\
\hline Coronary heart disease & $25(0.32)$ & $24(0.32)$ & 0.99 & $23(0.39)$ & $9(0.16)$ & 2.38 & $17(0.33)$ & $18(0.42)$ & 0.78 & -1.19 \\
\hline Stroke & $25(0.32)$ & $14(0.19)$ & 1.70 & $16(0.27)$ & $8(0.14)$ & 1.87 & $12(0.23)$ & $15(0.35)$ & 0.66 & -0.51 \\
\hline Venous thromboembolism & $27(0.34)$ & $14(0.19)$ & 1.84 & $16(0.27)$ & $6(0.11)$ & 2.49 & $12(0.23)$ & $11(0.26)$ & 0.90 & -2.45 \\
\hline Invasive breast cancer & $40(0.50)$ & $22(0.29)$ & 1.73 & $34(0.57)$ & $12(0.22)$ & 2.64 & $27(0.53)$ & $20(0.47)$ & 1.12 & 2.56 \\
\hline Endometrial cancer & $10(0.13)$ & $5(0.07)$ & 1.91 & $1(0.02)$ & $4(0.07)$ & 0.23 & $1(0.02)$ & $5(0.12)$ & 0.17 & -1.58 \\
\hline Colorectal cancer & $9(0.11)$ & $20(0.26)$ & 0.43 & $4(0.07)$ & $8(0.14)$ & 0.47 & $5(0.10)$ & $7(0.16)$ & 0.59 & -0.81 \\
\hline Hip fracture & $8(0.10)$ & $11(0.15)$ & 0.69 & $5(0.08)$ & $8(0.14)$ & 0.58 & $6(0.12)$ & $9(0.21)$ & 0.55 & 0.25 \\
\hline Total death & $55(0.69)$ & $48(0.63)$ & 1.09 & $41(0.69)$ & $44(0.79)$ & 0.87 & $44(0.86)$ & $44(1.04)$ & 0.83 & -0.79 \\
\hline Global index & 155 (1.96) & 127 (1.68) & 1.16 & 112 (1.88) & 77 (1.38) & 1.36 & 100 (1.95) & 99 (2.33) & 0.84 & -0.87 \\
\hline
\end{tabular}


affect the results. These analyses suggest that the intention-to-treat estimates of HRs may somewhat underestimate the effect sizes relative to what would be observed with full adherence to study medications.

\section{COMMENT}

The WHI provides evidence from a large randomized trial that addresses the important issue of whether most women with an intact uterus in the decades of life following menopause should consider hormone therapy to prevent chronic disease. The WHI enrolled a cohort of mostly healthy, ethnically diverse women, spanning a large age range (50-79 years at baseline). It is noteworthy that the increased risks for cardiovascular disease and invasive breast cancer were present across racial/ethnic and age strata and were not influenced by the antecedent risk status or prior disease. Hence, the results are likely to be generally applicable to healthy women in this age range. At the time the trial was stopped, the increases in numbers of invasive breast cancers, CHD, stroke, and PE made approximately equal contributions to harm in the estrogen plus progestin group compared with placebo, which were not counterbalanced by the smaller reductions in numbers of hip fractures and colorectal cancers.

\section{Cardiovascular Disease}

Even though the trial was stopped early for harm from breast cancer, a sufficient number of CHD events had occurred by 5.2 years of average follow-up to suggest that continuation to the planned end would have been unlikely to yield a favorable result for the primary outcome of CHD. Even if there were a reversal of direction toward benefit of a magnitude seen in the observational studies (ie, a risk reduction of $55 \%$ ) during the remaining years, conditional power analyses indicate that less than $10 \%$ power remained for showing potential benefit if the trial continued.

The WHI finding that estrogen plus progestin does not confer benefit for preventing CHD among women with a uterus concurs with HERS findings among women with clinically apparent $\mathrm{CHD},{ }^{10}$ with the Estrogen Replacement for Atherosclerosis trial, in which estrogen plus progestin did not inhibit progression, ${ }^{23}$ and with a trial in women with unstable angina that did not observe a reduction in ischemic events. ${ }^{24}$ The finding of an increased risk after initiation of treatment in WHI is similar to HERS. In HERS, after 4.1 and 6.8 years of follow-up, hormone therapy did not increase or decrease risk of cardiovascular events in women with CHD. ${ }^{25}$ The WHI extends these findings to include a wider range of women, including younger women and those without clinically apparent CHD, and indicates that the risk may persist for some years.

Unlike CHD, the excess risk of stroke in the estrogen plus progestin group was not present in the first year but appeared during the second year and persisted through the fifth year. Preliminary analyses indicate that the modest difference in blood pressure between groups does not contribute much to an explanation of the increase in strokes (data not shown). The findings in WHI for stroke are consistent with but somewhat more extreme than those of HERS, which reported a nonsignificant $23 \%$ increase in the treatment group. ${ }^{26}$ The results were also more extreme than those of the Women's Estrogen and Stroke Trial of estradiol (without progestin) in women with prior stroke, which found no effect of estrogen on recurrent strokes overall but some increase in the first 6 months. ${ }^{27}$ Trials of the effect of estradiol on carotid intima-media thickness have yielded conflicting results. ${ }^{28,29}$ At least 1 observational study has suggested that that use of estrogen plus progestin is associated with higher risk of stroke than estrogen alone. ${ }^{14}$ In WHI, there was no indication that excess strokes due to estrogen plus progestin were more likely to occur in older women, in women with prior stroke history, by race/ethnicity, or in women with high blood pressure at baseline. Therefore, it appears that estrogen plus progestin increases the risk of strokes in apparently healthy women.

Venous thromboembolism is an expected complication of postmenopausal hormones, and the pattern over time in WHI is consistent with the findings from HERS and several observational studies. ${ }^{30,31}$

\section{Cancer}

The WHI is the first randomized controlled trial to confirm that combined estrogen plus progestin does increase the risk of incident breast cancer and to quantify the degree of risk. The WHI could not address the risk of death due to breast cancer because with the relatively short follow-up time, few women in the WHI have thus far died as a result of breast cancer ( 3 in the active treatment group and 2 in the placebo group). The risk of breast cancer emerged several years after randomization. After an average follow-up of about 5 years, the adverse effect on breast cancer had crossed the monitoring boundary. The $26 \%$ excess of breast cancer is consistent with estimates from pooled epidemiological data, which reported a $15 \%$ increase for estrogen plus progestin use for less than 5 years and a 53\% increase for use for more than 5 years. ${ }^{32}$ It is also consistent with the (nonsignificant) $27 \%$ increase found after 6.8 years of follow-up in HERS. ${ }^{33}$

With more common use of estrogen plus progestin, several epidemiological studies have reported that estrogen plus progestin appears to be associated with greater risk of breast cancer than estrogen alone. ${ }^{34-37}$ In the PEPI trial, women in the 3 estrogen plus progestin groups had much greater increases in mammographic density (a predictor of breast cancer) than women in the estrogen or placebo groups ${ }^{38}$ In WHI, the HR for estrogen plus progestin was not higher in women with a family history or other risk factors for breast cancer, except for reported prior use of postmenopausal hormones. This may suggest a cumulative effect of years of exposure to postmenopausal hormones.

Endometrial cancer rates were low and were not increased by 5 years of es- 
trogen plus progestin exposure. Close monitoring for bleeding and treatment of hyperplasia may contribute to the absence of increased risk of endometrial cancer.

The reduction in colorectal cancer in the hormone group is consistent with observational studies, which have suggested fairly consistently that users of postmenopausal hormones may be at lower risk of colorectal cancer. ${ }^{39}$ The mechanisms by which hormone use might reduce risk are unclear. Results from other trials of postmenopausal hormones will help resolve the effects of hormones on colorectal cancer. ${ }^{40}$

\section{Fractures}

The reductions in clinical vertebral fractures, other osteoporotic fractures, and combined fractures supported the benefit for hip fractures found in this trial. These findings are consistent with the observational data and limited data from clinical trials ${ }^{41}$ and are also consistent with the known ability of estrogen (with or without progestin) to maintain bone mineral density. ${ }^{42}$ The WHI is the first trial with definitive data supporting the ability of postmenopausal hormones to prevent fractures at the hip, vertebrae, and other sites.

\section{Overall Risks and Benefits}

At the end of the trial, the global index indicated that there were more harmful than beneficial outcomes in the estrogen plus progestin group vs the placebo group. The monitored outcomes included in the global index were selected to represent diseases of serious import that estrogen plus progestin treatment might affect, and do not include a variety of other conditions and measures that may be affected in unfavorable or favorable ways (eg, gallbladder disease, diabetes, quality of life, and cognitive function). The data on these and other outcomes will be the subject of future publications. All-cause mortality was balanced between the groups; however, longer follow-up may be needed to assess the impact of the incident diseases on total mortality.
The absolute excess risk (or risk reduction) attributable to estrogen plus progestin was low. Over 1 year, 10000 women taking estrogen plus progestin compared with placebo might experience 7 more CHD events, 8 more strokes, 8 more PEs, 8 more invasive breast cancers, 6 fewer colorectal cancers, and 5 fewer hip fractures. Combining all the monitored outcomes, women taking estrogen plus progestin might expect 19 more events per year per 10000 women than women taking placebo. Over a longer period, more typical of the duration of treatment that would be needed to prevent chronic disease, the absolute numbers of excess outcomes would increase proportionately.

During the 5.2 years of this trial, the number of women experiencing a global index event was about 100 more per 10000 women taking estrogen plus progestin than taking placebo. If the current findings can be extrapolated to an even longer treatment duration, the absolute risks and benefits associated with estrogen plus progestin for each of these conditions could be substantial and on a population basis could account for tens of thousands of conditions caused, or prevented, by hormone use.

\section{Limitations}

This trial tested only 1 drug regimen, CEE, $0.625 \mathrm{mg} / \mathrm{d}$, plus MPA, $2.5 \mathrm{mg} / \mathrm{d}$, in postmenopausal women with an intact uterus. The results do not necessarily apply to lower dosages of these drugs, to other formulations of oral estrogens and progestins, or to estrogens and progestins administered through the transdermal route. It remains possible that transdermal estradiol with progesterone, which more closely mimics the normal physiology and metabolism of endogenous sex hormones, may provide a different risk-benefit profile. The WHI findings for CHD and VTE are supported by findings from HERS, but there is no other evidence from clinical trials for breast cancer and colorectal cancer, and only limited data from trials concerning fractures.

Importantly, this trial could not distinguish the effects of estrogen from those of progestin. The effects of progestin may be important for breast cancer and atherosclerotic diseases, including CHD and stroke. Per protocol, in a separate and adequately powered trial, WHI is testing the hypothesis of whether oral estrogen will prevent CHD in 10739 women who have had a hysterectomy. The monitoring of this trial is similar to that for the trial of estrogen plus progestin. At an average follow-up of 5.2 years, the DSMB has recommended that this trial continue because the balance of overall risks and benefits remains uncertain. These results are expected to be available in 2005, at the planned termination.

The relatively high rates of discontinuation in the active treatment arm (42\%) and crossover to active treatment in the placebo arm (10.7\%) are a limitation of the study; however, the lack of adherence would tend to decrease the observed treatment effects. Thus, the results presented here may underestimate the magnitude of both adverse effects on cardiovascular disease and breast cancer and the beneficial effects on fractures and colorectal cancer among women who adhere to treatment.

The fact that the trial was stopped early decreases the precision of estimates of long-term treatment effects. A longer intervention period might have shown more pronounced benefit for fractures and might have yielded a more precise test of the hypothesis that treatment reduces colorectal cancer. Nonetheless, it appears unlikely that benefit for CHD would have emerged by continuing the trial to its planned termination. The trial results indicate that treatment for up to 5.2 years is not beneficial overall and that there is early harm for $\mathrm{CHD}$, continuing harm for stroke and VTE, and increasing harm for breast cancer with increasing duration of treatment. This risk-benefit profile is not consistent with the requirements for a viable intervention for the primary prevention of chronic diseases.

\section{Implications}

The WHI trial results provide the first definitive data on which to base treat- 
ment recommendations for healthy postmenopausal women with an intact uterus. This trial did not address the short-term risks and benefits of hormones given for the treatment of menopausal symptoms. On the basis of HERS and other secondary prevention trials, the American Heart Association recommended against initiating postmenopausal hormones for the secondary prevention of cardiovascular disease. ${ }^{43}$ The American Heart Association made no firm recommendation for primary prevention while awaiting the results from randomized clinical trials such as WHI, and stated that continuation of the treatment should be considered on the basis of established noncoronary benefits and risks, possible coronary benefits and risks, and patient preference.

Results from WHI indicate that the combined postmenopausal hormones CEE, $0.625 \mathrm{mg} / \mathrm{d}$, plus MPA, $2.5 \mathrm{mg} / \mathrm{d}$, should not be initiated or continued for the primary prevention of CHD. In addition, the substantial risks for cardiovascular disease and breast cancer must be weighed against the benefit for fracture in selecting from the available agents to prevent osteoporosis.

Writing Group for the Women's Health Initiative Investigators: Jacques $\mathrm{E}$. Rossouw, MBChB, MD, National Heart, Lung, and Blood Institute, Bethesda, Md; Garnet L. Anderson, PhD, Ross L. Prentice, PhD, Andrea Z. LaCroix, PhD, and Charles Kooperberg, PhD, Fred Hutchinson Cancer Research Center, Seattle, Wash; Marcia L. Stefanick, PhD, Stanford University Clinical Center, Stanford, Calif; Rebecca D. Jackson, MD, Ohio State University Clinical Center, Columbus; Shirley A. A. Beresford, PhD, Fred Hutchinson Cancer Research Center, Seattle, Wash; Barbara V. Howard, PhD, MedStar Research Institute, Washington, DC; Karen C. Johnson, MD, MPH, University of Tennessee, Memphis; Jane Morley Kotchen, MD, Medical College of Wisconsin, Milwaukee; Judith Ockene, PhD, University of Massachusetts Medical School, Worcester.

Financial Disclosures: Dr LaCroix is an investigator on 2 osteoporosis studies separately funded by Merck and Pfizer. Dr Jackson is an investigator on 1 osteoporosis study funded by Merck and 1 hormone study on libido in women funded by Procter \& Gamble Pharmaceuticals.

For Correspondence: Jacques E. Rossouw, MBChB, $M D$, Division of Women's Health Initiative, National Heart, Lung, and Blood Institute, 6705 Rockledge Dr, One Rockledge Ctr, Suite 300, MS/7966, Bethesda, MD 20817 (e-mail: rossouw@nih.gov); Garnet L. Anderson, PhD, Division of Public Health Sciences, Fred Hutchinson Cancer Research Center, 1100 Fairview Ave N, MP-1002, PO Box 19024, Seattle, WA 98109-1024 (e-mail: garnet@whi.org).

Reprints: WHI Clinical Coordinating Center, Division of Public Health Sciences, Fred Hutchinson Cancer Research Center, 1100 Fairview Ave N, MP1002, PO Box 19024, Seattle, WA 98109-1024.
Author Contributions: Dr Anderson, as co-principal investigator of the Women's Health Initiative Clinical Coordinating Center, had full access to the data in the study and takes responsibility for the integrity of the data and the accuracy of the data analyses.

Study concept and design: Rossouw, Anderson, Prentice.

Acquisition of data: Anderson, Prentice, LaCroix, Kooperberg, Stefanick, Jackson, Beresford, Howard, Johnson, Kotchen, Ockene.

Analysis and interpretation of data: Rossouw, Anderson, Prentice, LaCroix, Kooperberg, Stefanick, Jackson. Drafting of the manuscript: Rossouw, Anderson, Prentice.

Critical revision of the manuscript for important intellectual content: Rossouw, Anderson, LaCroix, Kooperberg, Stefanick, Jackson, Beresford, Howard, Johnson, Kotchen, Ockene.

Statistical expertise: Anderson, Prentice, Kooperberg.

Obtained funding: Rossouw, Anderson, Prentice, Stefanick, Beresford, Howard, Kotchen, Ockene. Administrative, technical, or material support: Rossouw, Anderson, Prentice, LaCroix, Kooperberg, Stefanick, Jackson, Beresford, Howard, Johnson, Kotchen, Ockene.

Study supervision: Rossouw, Anderson, LaCroix, Stefanick, Jackson, Beresford, Howard, Johnson, Kotchen, Ockene.

WHI Steering Committee: Clinical Centers: Marcia L. Stefanick (chair), Stanford Center for Research in Disease Prevention, Stanford University; Rebecca D. Jackson (vice chair), Ohio State University; Catherine I. Allen, University of Wisconsin, Madison; Annlouise R. Assaf, Brown University, Providence, RI; Tamsen Bassford, University of Arizona, Tucson/Phoenix; Shirley A. A. Beresford, Fred Hutchinson Cancer Research Center; Henry Black, Rush-Presbyterian-St Luke's Medical Center, Chicago, Ill; Robert Brunner, University of Nevada, Reno; Gregory L. Burke, Wake Forest University School of Medicine, WinstonSalem, NC; Bette Caan, Kaiser Permanente Division of Research, Oakland, Calif; Rowan T. Chlebowski, Harbor-UCLA Research and Education Institute, Torrance, Calif; David Curb, University of Hawaii, Honolulu; Margery Gass, University of Cincinnati, Cincinnati, Ohio; Jennifer Hays, Baylor College of Medicine, Houston, Tex; Gerardo Heiss, University of North Carolina, Chapel Hill; Susan Hendrix, Wayne State University School of Medicine/Hutzel Hospital, Detroit, Mich; Barbara V. Howard, MedStar Research Institute, Washington, DC; Judith Hsia, George Washington University, Washington, DC; F. Allan Hubbell, University of California, Irvine, Orange; Karen C. Johnson, University of Tennessee, Memphis; Howard Judd, University of California, Los Angeles; Jane Morley Kotchen, Medical College of Wisconsin, Milwaukee; Lewis Kuller, University of Pittsburgh, Pittsburgh, Pa; Dorothy Lane, State University of New York at Stony Brook; Robert D. Langer, University of California, San Diego, LaJolla/Chula Vista; Norman Lasser, University of Medicine and Dentistry of New Jersey, Newark; Cora E. Lewis, University of Alabama at Birmingham; Marian Limacher, University of Florida, Gainesville/Jacksonville; JoAnn Manson, Brigham and Women's Hospital, Harvard Medical School, Boston, Mass; Karen Margolis, University of Minnesota, Minneapolis; Judith Ockene, University of Massachusetts Medical School, Worcester; Mary Jo O'Sullivan, University of Miami, Miami, Fla; Lawrence Phillips, Emory University, Atlanta, Ga; Cheryl Ritenbaugh, Kaiser Permanente Center for Health Research, Portland, Ore; John Robbins, University of California, Davis, Sacramento; Robert Schenken, University of Texas Health Science Center, San Antonio; Sylvia WassertheilSmoller, Albert Einstein College of Medicine, Bronx, NY: Maurizio Trevisan, State University of New York at Buffalo; Linda Van Horn, Northwestern Univer- sity, Chicago/Evanston, Ill; and Robert Wallace, University of lowa, lowa City/Davenport; Program Office: Jacques E. Rossouw, National Heart, Lung, and Blood Institute; Clinical Coordinating Center: Andrea Z. LaCroix, Ruth E. Patterson, and Ross L. Prentice, Fred Hutchinson Cancer Research Center. Data and Safety Monitoring Board: Janet Wittes (chair), Eugene Braunwald, Margaret Chesney, Harvey Cohen, Elizabeth Barrett-Connor, David DeMets, Leo Dunn, Johanna Dwyer, Robert P. Heaney, Victor Vogel, LeRoy Walters, and Salim Yusuf.

Funding/Support: The National Heart, Lung, and Blood Institute funds the WHI program. WyethAyerst Research provided the study medication (active and placebo).

Acknowledgment: The WHI Steering Committee gratefully acknowledges the dedicated efforts of the WHI participants and of key WHI investigators and staff at the clinical centers and the Clinical Coordinating Center. A full listing of the $\mathrm{WHI}$ investigators can be found at http://www.whi.org.

\section{REFERENCES}

1. The Women's Health Initiative Study Group. Design of the Women's Health Initiative clinical trial and observational study. Control Clin Trials. 1998;19: 61-109

2. Stampfer M, Colditz G. Estrogen replacement therapy and coronary heart disease: a quantitative assessment of the epidemiologic evidence. Prev Med. 1991;20:47-63.

3. Grady D, Rueben SB, Pettiti DB, et al. Hormone therapy to prevent disease and prolong life in postmenopausal women. Ann Intern Med. 1992;117: 1016-1037.

4. Rijpkema $A H$, van der Sanden AA, Ruijs AH. Effects of postmenopausal estrogen-progesterone therapy on serum lipids and lipoproteins: a review. Ma turitas. 1990;12:259-285.

5. Adams MR, Kaplan JR, Manuck SB, et al. Inhibition of coronary artery atherosclerosis by 17-beta estradiol in ovariectomized monkeys: lack of an effect of added progesterone. Arteriosclerosis. 1990;10: 1051-1057.

6. Weiss NS, Ure CL, Ballard JH, Williams AR, Daling $J R$. Decreased risk of fractures of the hip and lower forearm with postmenopausal use of estrogen. N Engl J Med. 1980;303:1195-1198.

7. Genant HK, Baylink DJ, Gallagher JC, Harris ST Steiger $\mathrm{P}$, Herber M. Effect of estrone sulfate on postmenopausal bone loss. Obstet Gynecol. 1990;76 579-584

8. Steinberg KA, Thacker SB, Smith SJ, et al. A metaanalysis of the effect of estrogen replacement therapy on the risk of breast cancer. JAMA. 1991;265:1985 1990.

9. Gerhardsson de VM, London S. Reproductive factors, exogenous female hormones, and colorecta cancer by subsite. Cancer Causes Control. 1992;3: 355-360.

10. Hulley S, Grady D, Bush T, et al. Randomized trial of estrogen plus progestin for secondary prevention of coronary heart disease in postmenopausal women. JAMA. 1998;280:605-613.

11. Grodstein F, Manson JE, Stampfer MJ. Postmenopausal hormone use and secondary prevention of coronary events in the Nurses' Health Study. Ann Intern Med. 2001;135:1-8.

12. Alexander KP, Newby LK, Hellkamp AS, et al. Initiation of hormone replacement therapy after acute myocardial infarction is associated with more cardiac events during follow-up. J Am Coll Cardiol. 2001; 38:1-7

13. Heckbert SR, Kaplan RC, Weiss NS, et al. Risk of recurrent coronary events in relation to use and recent initiation of postmenopausal hormone therapy. Arch Intern Med. 2001;161:1709-1173. 
14. Grodstein F, Manson JE, Colditz GA, Willit WC, Speizer FE, Stampfer MJ. A prospective, observational study of postmenopausal hormone therapy and primary prevention of cardiovascular disease. Ann Intern Med. 2000;133:933-941.

15. The Writing Group for the PEPI Trial. Effects of hormone replacement therapy on endometrial histology in postmenopausal women: the Postmenopausal Estrogen/Progestin Interventions (PEPI) Trial. JAMA. 1996;275:370-375.

16. Ives DG, Fitzpatrick AL, Bild DE, et al. Surveillance and ascertainment of cardiovascular events: the Cardiovascular Health Study. Ann Epidemiol. 1995; 5:275-285.

17. Cox DR. Regression analysis and life tables. $J R$ Stat Soc. 1972:34:187-220.

18. O'Brien PC, Fleming RT. A multiple testing procedure for clinical trials. Biometrics. 1979;35:549556.

19. Freedman LS, Anderson G, Kipnis V, et al. Approaches to monitoring the results of long-term disease prevention trials: examples from the Women's Health Initiative. Control Clin Trials. 1996;17:509525

20. Gail MH, Brinton LA, Byar DP, et al. Projecting individualized probabilities of developing breast cancer for white females who are being examined annually. J Natl Cancer Inst. 1989;81:1879-1886. 21. Pilon D, Castilloux AM, Lelorier J. Estrogen replacement therapy: determinants of persistence with treatment. Obstet Gynecol. 2001;97:97-100.

22. Writing Group for the PEPI Trial. Effects of estrogen or estrogen/progestin regimens on heart disease risk factors in postmenopausal women. JAMA. 1995 273:199-208.

23. Herrington DM, Reboussin DM, Brosnihan KB, et al. Effects of estrogen replacement on the progression of coronary artery atherosclerosis. N Eng/ J Med. 2000;343:522-529.

24. Schulman SP, Thiemann DR, Ouyang P, et al. Effects of acute hormone therapy on recurrent ischemia in postmenopausal women with unstable angina. J Am Coll Cardiol. 2002;39:231-237.
25. Grady D, Herrington D, Bittner V, et al, for the HERS Research Group. Cardiovascular disease outcomes during 6.8 years of hormone therapy: Heart and Estrogen/progestin Replacement Study Follow-up (HERS II). JAMA. 2002;288:49-57.

26. Simon JA, Hsia J, Cauley JA, et al. Postmenopausal hormone therapy and risk of stroke: the Heart and Estrogen-progestin Replacement Study (HERS). Circulation. 2001:103:638-642.

27. Viscoli CM, Brass LM, Kernan WN, Sarrel PM, Suissa S, Horwitz RI. A clinical trial of estrogenreplacement therapy after ischemic stroke. $N$ Engl J Med. 2001;345:1243-1249.

28. Hodis HN, Mack WJ, Lobo RA, et al. Estrogen in the prevention of atherosclerosis: a randomized, double-blind controlled trial. Ann Intern Med. 2001; 135:939-953.

29. Angerer $P$, Stork $S$, Kothny $W$, Schmitt $P$, von Schacky C. Effect of oral postmenopausal hormone replacement on progression of atherosclerosis: a randomized, controlled trial. Arterioscler Thromb Vasc Biol. 2001;21:262-268.

30. Castellsague J, Perez Gutthann S, Garcia Rodriguez LA. Recent epidemiological studies of the association between hormone replacement therapy and venous thromboembolism: a review. Drug Saf. 1998; 18:117-123.

31. Grady D, Wenger NK, Herrington D, et al. Postmenopausal hormone therapy increases risk for venous thromboembolic disease: the Heart and Estrogen/ progestin Replacement Study. Ann Intern Med. 2000; 132:689-696.

32. Collaborative Group on Hormonal Factors in Breast Cancer. Breast cancer and hormone replacement therapy: collaborative reanalysis of data from 51 epidemiological studies of 52,705 women with breast cancer and 108,411 women without breast cancer. Lancet. 1997:350:1047-1059.

33. Hulley S, Furberg C, Barrett-Connor E, et al, for the HERS Research Group. Noncardiovascular disease outcomes during 6.8 years of hormone therapy: Heart and Estrogen/progestin Replacement Study Follow-up (HERS II). JAMA. 2002;288:58-66.
34. Schairer C, Lubin J, Troisi R, Sturgeon S, Brinton L, Hoover R. Menopausal estrogen and estrogenprogestin replacement therapy and breast cancer risk JAMA. 2000;283:485-491.

35. Ross RK, Paganini-Hill A, Wan PC, Pike MC. Effect of hormone replacement therapy on breast cancer risk: estrogen versus estrogen plus progestin. J Natl Cancer Inst. 2000;92:328-332.

36. Colditz GA, Hankinson SE, Hunter DJ, et al. The use of estrogens and progestins and the risk of breast cancer in postmenopausal women. N Eng/ J Med. 1995; 332:1589-1593.

37. Magnusson C, Baron JA, Correia N, Bergstrom R Adami H-O, Persson I. Breast-cancer risk following long-term oestrogen and oestrogen-progestinreplacement therapy. Int J Cancer. 1999;81:339 344.

38. Greendale GA, Reboussin BA, Sie A, et al. Effects of estrogen and estrogen-progestin on mammo graphic parenchymal density. Ann Intern Med. 1999, 130:262-269.

39. Grodstein F, Newcomb PA, Stampfer MJ. Postmenopausal hormone therapy and the risk of colorectal cancer: a review and meta-analysis. Am J Med. 1999;106:574-582

40. Vickers MR, Meade TW, Wilkes HC. Hormone replacement therapy and cardiovascular disease: the case for a randomized controlled trial. Ciba Found Symp. 1995;191:150-160.

41. Torgerson DJ, Bell-Seyer SE. Hormone replacement therapy and prevention of nonvertebral fractures: a meta-analysis of randomized trials. JAMA 2001;285:2891-2897.

42. Writing Group for the PEPI Trial. Effects of hormone therapy on bone mineral density: results from the Postmenopausal Estrogen/Progestin Interventions (PEPI) Trial. JAMA. 1996;275:1389. 1396.

43. Mosca L, Collins P, Herrington DM, et al. Hor mone replacement therapy and cardiovascular disease: a statement for healthcare professionals from the American Heart Association. Circulation. 2001;104: 499-503. 